\title{
BMJ Open Sepsis and Critical Illness Research Center investigators: protocols and standard operating procedures for a prospective cohort study of sepsis in critically ill surgical patients
}

Tyler J Loftus, ${ }^{1,2}$ Juan C Mira, ${ }^{1,2}$ Tezcan Ozrazgat-Baslanti, ${ }^{1,3}$ Gabriella L Ghita, ${ }^{4}$ Zhongkai Wang, ${ }^{4}$ Julie A Stortz, ${ }^{1,2}$ Babette A Brumback, ${ }^{1,4}$ Azra Bihorac, ${ }^{1,3}$ Mark S Segal, ${ }^{1,5}$ Stephen D Anton, ${ }^{1,6}$ Christiaan Leeuwenburgh, ${ }^{1,6}$ Alicia M Mohr, ${ }^{1,2}$ Philip A Efron, ${ }^{1,2}$ Lyle L Moldawer, ${ }^{1,2}$ Frederick A Moore, ${ }^{1,2}$ Scott C Brakenridge ${ }^{1,2}$

To cite: Loftus TJ, Mira JC, Ozrazgat-Baslanti T, et al. Sepsis and Critical IIIness Research Center investigators: protocols and standard operating procedures for a prospective cohort study of sepsis in critically ill surgical patients. BMJ Open 2017;7:e015136. doi:10.1136/ bmjopen-2016-015136

- Prepublication history and additional material are available. To view these files please visit the journal online (http://dx.doi. org/10.1136/bmjopen-2016015136).

Received 10 November 2016 Revised 12 May 2017 Accepted 30 June 2017
CrossMark

For numbered affiliations see end of article.

Correspondence to Dr Scott C Brakenridge; Scott.Brakenridge@surgery. ufl.edu

\section{ABSTRACT}

Introduction Sepsis is a common, costly and morbid cause of critical illness in trauma and surgical patients. Ongoing advances in sepsis resuscitation and critical care support strategies have led to improved in-hospital mortality. However, these patients now survive to enter state of chronic critical illness (CCI), persistent low-grade organ dysfunction and poor long-term outcomes driven by the persistent inflammation, immunosuppression and catabolism syndrome (PICS). The Sepsis and Critical Illness Research Center (SCIRC) was created to provide a platform by which the prevalence and pathogenesis of $\mathrm{CCl}$ and PICS may be understood at a mechanistic level across multiple medical disciplines, leading to the development of novel management strategies and targeted therapies. Methods Here, we describe the design, study cohort and standard operating procedures used in the prospective study of human sepsis at a level 1 trauma centre and tertiary care hospital providing care for over 2600 critically ill patients annually. These procedures include implementation of an automated sepsis surveillance initiative, augmentation of clinical decisions with a computerised sepsis protocol, strategies for direct exportation of quality-filtered data from the electronic medical record to a research database and robust longterm follow-up.

Ethics and dissemination This study has been registered at ClinicalTrials.gov, approved by the University of Florida Institutional Review Board and is actively enrolling subjects. Dissemination of results is forthcoming.

\section{INTRODUCTION}

Sepsis as both a cause and a complication of surgical intensive care unit (ICU) admission is common, costly and morbid. Hospitalisation with sepsis has become more common than hospitalisation with myocardial infarction, with annual costs over US $\$ 20$ billion in the USA. ${ }^{12}$ Mortality rates for sepsis range

\section{Strengths and limitations of this study}

- Computerised decision support will minimise the influence of variability in management practices.

- Robust long-term follow-up will allow for deeper understanding of functional recovery following sepsis.

- Investigation of targeted therapies is currently limited by deficiencies in our understanding of sepsis pathophysiology.

from $18 \%-28 \%$, and remain unacceptably high despite more than 30 years of intensive research. ${ }^{34}$ Recent advances in resuscitation strategies have improved in-hospital mortality, but sepsis survivors often enter a state of chronic critical illness (CCI) driven by the persistent inflammation, immunosuppression and catabolism syndrome (PICS) ${ }^{56}$ (figure 1). However, the pathophysiology of these conditions remains incompletely understood. The Sepsis and Critical Illness Research Center (SCIRC) was created to provide a platform to better understand the pathophysiology of PICS. The objective of this prospective cohort study of sepsis in critically ill surgical patients is to understand the prevalence and pathogenesis of PICS at a mechanistic level across multiple medical disciplines, leading to the development of novel management strategies and targeted therapies.

\section{METHODS AND ANALYSIS}

\section{Setting}

The University of Florida (UF) Health Shands Hospital (Gainesville, Florida; U.S.A.) is a level 1 trauma and tertiary care centre 


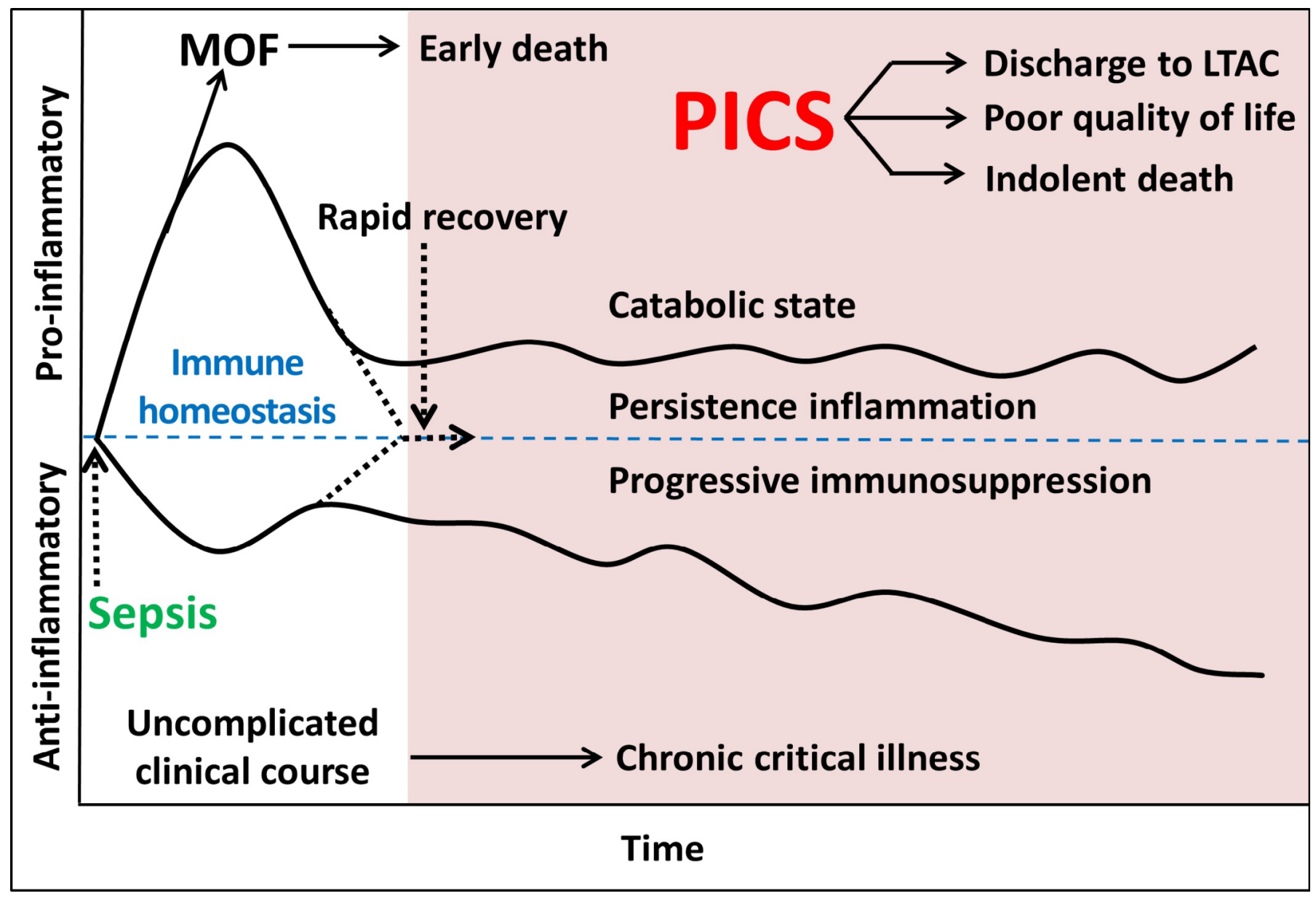

Figure 1 PICS, adapted from Rosenthal et $a l^{6}$ and Mira et al. ${ }^{47} \mathrm{CARS}$, compensatory anti-inflammatory response syndrome; LTAC, long-term acute care facility; MOF, multiple organ failure; PICS, persistent inflammation, immunosuppression and catabolism syndrome; SIRS, systemic inflammatory response syndrome.

with two trauma/surgical ICUs totalling 48 beds, which serve as the recruitment base for this cohort study. UF Health is the sole tertiary care centre for a greater than 90 mile radius catchment area, including over 1.5 million people. Together, the trauma and surgical ICU teams manage over 2600 critically ill patients annually. Each ICU has a dedicated surgical critical care team including a board certified attending intensivist, critical care fellows, surgical and anaesthesia residents, and advanced practice providers (physician assistants and nurse practitioners). These teams collaborate with unit-dedicated pharmacists, respiratory therapists, physical therapists, occupational therapists, nutritionists and social workers. Board certified attending acute care surgeons and critical care fellows provide in-hospital coverage 24 hours per day, 7 days per week.

\section{Study design and population}

This is a prospective, longitudinal cohort study of surgical ICU patients that develop sepsis. Based on preliminary data and a priori power analyses, 400 patients will be enrolled over a period of 4 years, with subsequent 12-month individual follow-up. Enrolment began in January 2015 and will continue through January 2019, and beyond if funding permits. Inclusion criteria are presence in the trauma/surgical ICU, age $\geq 18$ years, and diagnosis with sepsis, severe sepsis or septic shock with subsequent initiation of the computerised clinical decision support (CCDS)-directed sepsis protocol. ${ }^{7-9}$ Septic patients are initially identified by a modified version of the Modified Early Warning Score (MEWS-SRS), ${ }^{10}$ which screens for sepsis based on temperature, heart rate, respiratory rate, blood pressure and level of consciousness (table 1). In the emergency department and on surgical wards, this score is calculated for each patient on arrival, every time vital signs are recorded, and any time a patient has an acute change from their baseline physiologic status, prompting further investigation. Patients identified by the MEWS screening protocol are then directly assessed by a physician or advance practice provider for bedside clinical adjudication of the presence of sepsis (a systemic inflammatory response with a source of infection), severe sepsis (sepsis-induced tissue hypoperfusion or organ dysfunction), or septic shock (severe sepsis with persistent arterial hypotension despite volume resuscitation) based on consensus definitions. ${ }^{11-14}$ This screening and diagnostic process has been automated 
Table 1 The Modified Early Warning Signs (MEWS)-Sepsis Recognition Score (SRS) grading scale, adapted from Croft et al, ${ }^{7}$ was used as a screening tool to identify patients who may be developing sepsis

\begin{tabular}{llllllll}
\hline Points & $\mathbf{3}$ & $\mathbf{2}$ & $\mathbf{1}$ & $\mathbf{0}$ & $\mathbf{1}$ & $\mathbf{2}$ & $\mathbf{3}$ \\
\hline Temp & $<32$ & $<35$ & $<36$ & $36.0-38.4$ & $38.5-38.9$ & $39.0-40.9$ & $\geq 41$ \\
HR & $<40$ & $40-44$ & $45-50$ & $51-100$ & $101-110$ & $111-129$ & $\geq 129$ \\
RR & $\leq 7$ & 8 & 9 & $10-14$ & $15-20$ & $21-29$ & $\geq 30$ \\
SBP & $\leq 70$ & $71-80$ & $81-100$ & $101-160$ & $161-180$ & $181-199$ & $\geq 200$ \\
Mental & Unresponsive & Responds to & Responds to & Alert, & Mildly agitated, Very agitated, Extremely \\
status* & & noxious stimuli & voice or tap & cooperative & confused & $\begin{array}{l}\text { requires } \\
\text { restraints }\end{array}$ \\
WBC & $<1.0 \dagger$ & $1.0-2.9 \dagger$ & - & $3.0-14.9$ & $15.0-19.9$ & $20.0-39.9$ & to self or others \\
\hline
\end{tabular}

${ }^{*}$ Do not score if the patient is receiving a sedating medication or has a general medical condition affecting mental status (eg, traumatic brain injury, stroke).

†Do not score if the patient is receiving oncolytic therapy.

Temp, temperature $\left({ }^{\circ} \mathrm{C}\right), \mathrm{HR}$, heart rate; RR, respiratory rate; SBP, systolic blood pressure $(\mathrm{mm} \mathrm{Hg})$; WBC, white cell count $\left(\mathrm{x} 10^{9} / \mathrm{L}\right)$.

The provider is notified if the patient has a total score $\geq 6$, three points in any single category, worsening mental status or an increase in fraction of inspired oxygen $\left(\mathrm{FiO}_{2}\right)$.

and embedded within the UF Health electronic medical record (Epic Systems, Verona, WI, USA). All cases that are deemed to have sepsis, severe sepsis or septic shock by the physician or advanced practice provider at the bedside are then reviewed in detail by a faculty member of the SCIRC to ensure that the diagnosis was appropriate, and are reviewed again at weekly SCIRC sepsis adjudication meetings for the same purpose.

Exclusion criteria are age $<18$ years, severe traumatic brain injury (ie, CT evidence of neurological injury and Glasgow Coma Scale score $<8$ ), spinal cord injury resulting in permanent sensory and/or motor deficits, sepsis with an uncontrollable source (eg, unresectable bowel ischaemia), New York Heart Association class IV heart failure, Child-Pugh class B or C liver disease, known HIV infection with CD4 count $<200$ cells $/ \mathrm{mm}^{3}$, organ transplant recipient on an immunosuppressant agent, chemotherapy or radiotherapy within 30 days prior to onset of sepsis, expected lifespan $<3$ months due to severe pre-existing comorbidities, active Do Not Resuscitate or Do Not Intubate order, pregnancy, incarceration or institutionalisation. Demographics, comorbidities, illness severity, length of stay and discharge disposition for patients who have been enrolled in the study are listed in table 2 .

Within the study population, cohort analyses will include comparisons between patients who develop CCI versus patients who experience early recovery from sepsis. Among CCI patients, patients who develop PICS will be compared with patients who do not. In addition, inflammatory, immunosuppression and catabolism biomarkers will be measured in age-matched healthy control for comparison to CCI, non-CCI and PICS patients.

\section{CCDS sepsis protocol}

Patients who are diagnosed with sepsis, severe sepsis or septic shock are started on a CCDS protocol, as previously described. ${ }^{7}$ This system was modified from a sepsis management protocol originally implemented at the Methodist Hospital in Houston, Texas. ${ }^{8}$ In brief, mobile bedside computer workstations were programmed with sepsis protocol algorithm logic that interacts with the patient and clinician by mapping clinical workflow and recommendations to patient physiology and clinical interventions. The sepsis protocol algorithm logic was developed by a multidisciplinary team of surgeons, intensivists, advanced practitioners, nurses, respiratory therapists, pharmacists, pathologists and computer engineers, based on Surviving Sepsis Campaign guidelines. ${ }^{14}$ The algorithm produces a recommendation; the clinician may accept or modify the recommendation, tailoring care to individual patient-specific factors. The selected intervention is then imputed, and the computerised clinical workflow and recommendations continue to evolve.

The computerised sepsis protocol is the platform for clinical decisions regarding initial volume resuscitation and antibiotic therapy initiation. For all other clinical decisions, the SCIRC developed protocols based on standard operating procedures from the Inflammation and the Host Response to Injury Collaborative Research Programme. ${ }^{15-24}$ The SCIRC protocols include a daily spontaneous breathing trial protocol (online supplementary figure 1), delirium protocol (online supplementary table 1), ${ }^{25}{ }^{26}$ product transfusion protocol (table 3) and a nutritional support protocol (online supplementary table 2). ${ }^{27}$

\section{Subject recruitment}

When a patient is diagnosed with sepsis, a page notification is sent to a team of research nurses who respond to evaluate for study enrolment 24 hours per day, 7 days per week. The research nurse on-call evaluates inclusion and exclusion criteria. If the patient qualifies, the research nurse seeks to obtain informed consent from the patient (if able) or legally authorised representative. Similar to the Inflammation and Host Response to Injury programme, a 96 hours deferral of informed consent exists for initial sample and data collection based on previous precedent at this institution, and approved by the institutional 
Table 2 Characteristics of enrolled patients

\begin{tabular}{|c|c|}
\hline Demographics & All patients $n=216$ \\
\hline Age in years, mean (SD) & $59.3(15.2)$ \\
\hline Male, n (\%) & $116(53.7)$ \\
\hline \multicolumn{2}{|l|}{ Race, n (\%) } \\
\hline Caucasian (White) & $191(88.4)$ \\
\hline African American & $20(9.3)$ \\
\hline Asian & $1(0.5)$ \\
\hline Pacific Islander & $0(0)$ \\
\hline Other & $3(1.4)$ \\
\hline BMI, median (25th, 75th) & $29.3(24.8,35.8)$ \\
\hline $\begin{array}{l}\text { Charlson Comorbidity Index, mean } \\
\text { (SD) }\end{array}$ & $4.3(3.0)$ \\
\hline $\begin{array}{l}\text { APACHE II score ( } 24 \text { hours), mean } \\
\text { (SD) }\end{array}$ & $18.0(8.1)$ \\
\hline Interfacility hospital transfer, n (\%) & $95(44.0)$ \\
\hline Hospital-acquired sepsis*, n (\%) & $88(40.7)$ \\
\hline ICU LOS, median (25th, 75th) & $7(3.5,18)$ \\
\hline Hospital LOS, median (25th, 75th) & $17(8,29)$ \\
\hline \multicolumn{2}{|l|}{ Discharge disposition, n (\%) } \\
\hline 'Good’ disposition & $117(54.2)$ \\
\hline Home & 38 (17.6) \\
\hline Home healthcare services & $69(31.2)$ \\
\hline Rehab & $10(4.6)$ \\
\hline 'Poor' disposition & $99(45.8)$ \\
\hline Long-term acute care facility & $30(13.9)$ \\
\hline Skilled nursing facility & $37(17.1)$ \\
\hline Another Hospital & $9(4.2)$ \\
\hline Hospice & 7 (3.2) \\
\hline Death & $16(7.4)$ \\
\hline
\end{tabular}

*Sepsis onset $\geq 48$ hours after hospital admission.

APACHE, Acute Physiology and Chronic Health Evaluation; BMI, body mass index; ICU, intensive care unit; LOS, length of stay.

review board (IRB).$^{15-17}$ If consent is not obtained within 96 hours, all initial blood samples and patient data are destroyed. If consent is initially obtained from the legally authorised representative and the patient regains decision-making capacity, the patient has the opportunity to withdraw from the study at that time. Study subjects may choose to participate during hospital admission with or without long-term follow-up, though long-term follow-up is encouraged, per study objectives.

Within the first 7 days, all study subjects undergo prospective clinical adjudication to confirm proper diagnosis, source identification and severity classification of sepsis during weekly SCIRC adjudication and retention committee meetings. As this study was designed and initiated prior to the Sepsis-3 consensus statement, sepsis severity is classified by previously established consensus definitions. ${ }^{11-14}$ However, data collected during the course of the study will allow for subsequent classification
Table 3 Indications for blood product transfusion

\begin{tabular}{ll}
\hline Blood product & Indications for transfusion \\
\hline Red blood cells & $\mathrm{Hb}<7 \mathrm{~g} / \mathrm{dL}$ or $\mathrm{HCT}<21 \%$ \\
& $\begin{array}{l}\mathrm{Hb}<10 \mathrm{~g} / \mathrm{dL} \text { or } \mathrm{HCT}<30 \% \text { and } \\
\text { symptomatic cardiovascular disease } \\
\text { Acute blood loss }>30 \% \text { of total blood } \\
\text { volume }\end{array}$ \\
Plasma (minimum & $\begin{array}{l}\text { INR }>1.7 \text { and active bleeding or } \\
\text { immediately prior to a procedure }\end{array}$ \\
& $\begin{array}{l}\text { Thrombotic thrombocytopenic purpura } \\
\text { fo-20 mL/kg) }\end{array}$ \\
& $\begin{array}{l}\text { Factor deficiency for which no specific } \\
\text { concentrate is available }\end{array}$ \\
& Fibrinogen $<100$ mg/dL \\
Cryoprecipitate & Factor XIII deficiency \\
& Perioperative management
\end{tabular}

$\mathrm{Hb}$, haemoglobin $\mathrm{HCT}$, haematocrit; INR, international normalised ratio.

and comparison to the subsequently released Sepsis-3 consensus statement, including assessment of qSOFA scores. $^{2829}$

\section{Data procurement and management}

Data describing baseline characteristics, management and outcomes for each study subject are prospectively collected, recorded and managed using the REDCap (REDCap consortium; www.projectredcap.org) research electronic case report form platform. ${ }^{30}$ In collaboration with the University of Florida Clinical and Translational Science Institute (CTSI), our centre developed an automated data collection and integration system that extracts clinical data from the electronic medical record and uploads the data to REDCap over a secure server on a daily basis. Raw data from the electronic medical record (EMR), including information on patient laboratory results, vital signs, medications and information related to hospital and ICU admission and discharge, are directly uploaded to the SCIRC database by the University of Florida Health Integrated Data Repository (IDR). The SCIRC database includes over 800 data fields describing the vital signs, clinical signs and symptoms of sepsis, medical history, laboratory values, microbiological analyses, cardiopulmonary resuscitation parameters, procedural interventions, medications, infusions and outcomes for each patient. Parameters available in the EMR are transferred to the SCIRC database after compilation and quality filtering via the IDR system. Data from biologic sampling analyses performed by the Bioanalytical Core (eg, flow cytometry, ELISA, multiplex, and gene analyses) are also transferred to the SCIRC drive as they are completed. The SCIRC database gives each project access to its own protected folder and to a bridge folder in which data can be placed for transfer to the Database Management and Biostatistics Core personnel for quality control and statistical analyses. The quality and accuracy of the transferred data is validated at regular intervals by 
the Database Management and Biostatistics Core by identifying potential outlier values and reviewing the source data with SCIRC faculty. Parameters that are not available in the electronic medical record are manually extracted and entered into the REDCap case report form. Data regarding the inpatient hospital course prior to protocol initiation are also collected. For patients transferred from another facility, this includes records from the outside facility regarding the initial signs and symptoms of sepsis, microbiological findings, antibiotic administration parameters and source control procedures.

\section{Biomarker sampling, processing and analysis}

Tissue samples are collected at scheduled intervals for biomarkers analyses of inflammation (eg, plasma tumour necrosis factor-alpha, interleukin (IL)-1, IL-2, IL-3, IL-5, IL-6, IL-10, IL-12, interferon-gamma, macrophage inflammatory protein 1-alpha), immunosuppression (eg, granulocytic and monocytic myeloid-derived suppressor cells in whole blood, expression of PD- ${ }^{31}$ and PDL-1 on blood monocytes and $\mathrm{CD}_{6} 6 \mathrm{~b}^{+}$neutrophils) and catabolism (eg, serum prealbumin, urine 3-methylhistidine, skeletal muscle high-resolution respirometry in situ, assessment of muscle morphology and myosin/actin ratio by histochemistry, and measurement of FoxO3A, MuRF1, MAFBx, BNIP, calpains, and 20S proteasome activity) from 12 hours out to 42 days or inpatient discharge for non-muscle samples. Muscle samples are obtained 28 days after sepsis protocol initiation. Skeletal muscle samples weighing $150-250 \mathrm{mg}$ are obtained from the vastus lateralis at the midpoint between the patella and the greater trochanter of the femur by trained practitioners using sterile technique under local anaesthesia, as previously described. ${ }^{32}$ A portion is immediately permeabilised for high resolution respiration measurements, a portion is mounted in embedding medium and frozen in isopentane for histochemical analysis, and the remaining tissue is frozen in liquid nitrogen and stored at $-80^{\circ} \mathrm{C}$.

For purposes of sample collection, time zero coincides with initiation of the sepsis protocol. Blood samples and laboratory measurements are obtained at the following time points, relative to sepsis protocol and study initiation: 12 hours, 1 day, 4 days, 7 days, 14 days, 21 days, 28 days, 35 days and 42 days. Initial sample processing, including centrifugation, labelling and freezing of patient samples, is performed 24 hours per day, 7 days per week at an on-site sample processing laboratory located within the trauma ICU. This laboratory contains a $-80^{\circ}$ freezer, a microfuge, a refrigerated centrifuge and an environmental hood. Flow cytometry is performed on fresh samples, and all other samples are stored in the $-80^{\circ}$ freezer, and subsequently transported to the Bioanalytics core or individual project laboratories as appropriate. Collected specimens are annotated, labelled and stored according to best-practice guidelines. ${ }^{33}$ Stored samples are maintained in a biobank that will remain available for future testing. Standard training is provided to all laboratory staff regarding machine calibration, sample processing, operator safety and quality control. Serum and plasma samples are collected in a fully filled collection tube, inverted 5-10 times and then maintained in a closed tube in vertical position until centrifugation. Samples are analysed in parallel with reagents from the same batch by the same technician. Analytic methods include flow cytometry, ELISA, multiplex and gene expression array (NanoString Technologies, Seattle, WA, USA and Affymetrix, Cleveland, OH, USA). The analytic plan followed the STROBE recommendations for observational cohort studies. ${ }^{34}$

\section{Subject retention, clinical assessments and long-term follow-} up

During the index hospitalisation, clinical assessments focus on host factors (eg, age, gender, comorbidities, hospital course prior to ICU admission), infection characteristics (eg, presumed type of infection, microbiological data, antibiotic therapy), sepsis severity (eg, haemodynamic parameters, vasopressor support, laboratory measures of hypoxaemia and tissue ischaemia, Sequential Organ Failure Assessment (SOFA) scores, ${ }^{35}$ APACHE II scores ${ }^{36}$ ), volume status by protocolised bedside echocardiography, procedural interventions to obtain source control, nutritional parameters (eg, nutrition provided by gastric, postpyloric and parenteral routes, weekly caloric and protein goals vs actual calories and protein administered, 24 hours urine collection to assess nitrogen balance, indirect calorimetry, and changes in body mass index and ideal body weight) and short-term outcomes (eg, infectious complications, non-infectious complications, ICU length of stay, days on mechanical ventilation, change in SOFA score over time, in-hospital mortality, discharge disposition).

Bedside echocardiography is performed by the transesophageal approach for intubated patients with an intact oesophagus and stomach, no known or suspected gastro-oesophageal variceal disease, and low risk for pathologically increased intracranial pressures. Echocardiography is performed by the transthoracic approach for all other patients. Assessments include the presence of pericardial fluid, characterisation of the right ventricle size as normal, collapsible or enlarged, characterisation of right and left ventricle contractility as normal, poor or hyperdynamic, description of the superior vena cava collapsibility index as less than or equal to $36 \%$, quantification of fractional area change (the difference in left ventricular area at end-diastole versus end-systole, divided by end-diastolic area), an interpretation of the findings as representing euvolemia, hypovolaemia or hypervolaemia, and a plan to start, discontinue, increase or decrease intravenous fluid therapy, vasopressor therapy and inotrope therapy based on echocardiography findings.

A retention committee creates an individualised follow-up plan for each study subject prior to discharge from the hospital. The retention committee meets twice per week to discuss all active study subjects, with special attention to subjects for whom long-term follow-up may be jeopardised by geographic and social impediments. 
Phone contact encounters are scheduled and used to predict retention problems. Parking and transportation costs are provided to the study subjects to maximise access to the research centre. Members of the retention committee are trained to recognise and address psychosocial issues and provide emotional support as needed. When medical and/or mental health problems necessitating further treatment are identified, the retention committee provides referrals to the appropriate specialists and ensures that care is provided in a timely fashion.

Long-term follow-up outpatient clinic visits occur at 3 months, 6 months and 12 months at the facilities of the University of Florida Institute on Aging. Clinical assessments at these time points will focus on functional recovery from sepsis by performing a battery of tests including the Rand 36-Item SF health Survey, ${ }^{37}$ Mini Nutritional Assessment, ${ }^{38}$ EQ-5D-3L Health Questionnaire, ${ }^{39}$ Hopkins Verbal Learning Test, ${ }^{40}$ Controlled Oral Word Association test, ${ }^{41}$ Modified Mini-Mental State Exam, ${ }^{42}$ ECOG/WHO/Zubrod score,${ }^{43}$ Short Physical Performance Battery, ${ }^{44}$ hand grip strength measurement and body composition measurements with the BIA 450 bioimpedance analyser (Biodynamics, Shoreline, WA, USA). If patients are unwilling or unable to return for outpatient clinical follow-up, home-visits are scheduled (up to 2-hour drive radius) or subjective data are collected via telephone interview.

To identify and evaluate the progression of sarcopenia, we will perform CT morphometric assessments of psoas and abdominal wall lean muscle mass at baseline, 3 months and 12 months, using SliceOmatic software (V.5.0 rev 6a; Tomovision, Magog, Quebec, Canada). Standard of care CT scans of the abdomen and pelvis conducted for diagnostic purposes while the patient was hospitalised were used for this analysis. Two of these CT scans were used: the baseline scan was performed within 3 days of sepsis protocol onset; the second scan was performed within 7 to 14 days of the baseline scan. To calculate the total skeletal muscle cross-sectional area $\left(\mathrm{cm}^{2}\right)$, trained investigators identified and quantified all skeletal muscles (psoas, paraspinal and abdominal wall muscles) at the level of the third lumbar (L3) vertebra where both transverse processes were visualised using established Hounsfield unit (-29 to 150) attenuation thresholds for skeletal muscle tissue..$^{45}$ The L3 vertebral level was chosen because skeletal muscle visualised at this axial plane has been shown to correlate with whole-body muscle mass. ${ }^{46}$ Skeletal muscle index $\left(\mathrm{SMI}, \mathrm{cm}^{2} / \mathrm{m}^{2}\right)$ was then calculated by normalising the total skeletal muscle cross-sectional area $\left(\mathrm{cm}^{2}\right)$ to patient height squared $\left(\mathrm{m}^{2}\right)$. Psoas muscle index (PMI, $\mathrm{cm}^{2} / \mathrm{m}^{2}$ ) was also calculated by normalising only the psoas muscle cross-sectional area $\left(\mathrm{cm}^{2}\right)$ to patient height squared $\left(\mathrm{m}^{2}\right)$.

\section{Outcome definitions and analytic design}

The primary outcomes of interest are the development of CCI and 1-year mortality rates after the development of sepsis. The CCI is defined as an extended course of critical illness with persistent organ dysfunction requiring intensive care resources. Extended course of illness requiring intensive care resources is defined as total ICU days $>14$ days or being discharged to another hospital, long-term acute care hospital, or hospice. Persistent organ dysfunction is defined as having a SOFA score of at least two in any organ system with the exception of at least one for cardiovascular system on day 14 in ICU after protocol onset or last SOFA score available, whichever comes first. Subjects are deemed to have developed CCI if they are discharged to dispositions associated with poor outcomes (eg, longterm acute care facility, skilled nursing facility) prior to ICU day 14 with ongoing evidence of organ dysfunction, as described above. Mortality at 1 year will be determined by prospective follow-up, or from the United States Social Security Death Index for those lost to follow-up.

Secondary outcomes of interest include changes in health, function and quality of life assessments at 1 year after sepsis onset. Analyses will include the development of biomarker and clinical prediction models for the development of CCI, as well as prediction models and the development of a 'CCI score' at ICU day 14 to predict 1 year mortality and poor functional outcomes. Additionally, biomarker analyses at day 14 will seek to characterise the presence of persistent inflammation, immunosuppression and catabolism in subjects who have developed CCI, consistent with the PICS pathophysiologic phenotype.

\section{ETHICS AND DISSEMINATION}

This study has been registered at ClinicalTrials.gov (NCT02276417). The University of Florida IRB approved this study. This work was supported by P50 GM111152-01 awarded by the National Institute of General Medical Sciences (NIGMS). TJL, JCM and JAS were supported by a post-graduate training grant (T32 GM-08721) in burns, trauma and perioperative injury from the NIGMS. The authors have read and understood BMJ policy on declaration of interests and declare that the authors have no competing interests. All investigators will complete annual training modules regarding the ethical conduct of research and Health Insurance Portability and Accountability Act (HIPAA) compliance, per IRB requirements. All investigators also complete National Institutes of Health conflict of interest disclosure training. Results will be presented at national and international conferences and reported in peer-reviewed journals. Dissemination of preliminary results is forthcoming.

\section{Summary}

Better strategies are needed to improve care for millions of critically ill patients with sepsis and septic shock. While in-hospital mortality has decreased, a new phenotype of CCI driven by PICS physiology has emerged and appears to be associated with a substantial burden of morbidity and late mortality. Therefore, further investigation is needed to elucidate pathophysiology and identify therapeutic 
approaches for CCI and PICS. Through prospective multidisciplinary investigation augmented by automated sepsis surveillance, clinical decision support with a computerised sepsis protocol, advanced data management strategies, and robust long-term follow-up, the SCIRC seeks to develop novel management strategies and targeted therapies for critically ill septic patients.

\section{Author affiliations}

${ }^{1}$ Department of Surgery, Sepsis and Critical Illness Research Center in Gainesville, University of Florida Health, Gainesville, Florida, USA

${ }^{2}$ Department of Surgery, University of Florida Health, Gainesville, Florida, USA ${ }^{3}$ Department of Anesthesiology, University of Florida Health, Gainesville, Florida, USA ${ }^{4}$ Department of Biostatistics, University of Florida Health, Gainesville, Florida, USA ${ }^{5}$ Department of Medicine, University of Florida Health, Gainesville, Florida, USA ${ }^{6}$ Institute on Aging, University of Florida Health, Gainesville, Florida, USA

\section{Twitter @brakenridge_md}

Acknowledgements The authors would like to acknowledge the invaluable contributions of the UF Sepsis and Critical IIIness Research Center staff, including Jennifer Lanz, Ruth Davis, Jillianne Brakenridge, AshleyMcCray, Bridget Baisden, Ricky Ungaro, Dina Nacionales, Marvin Dirain, Tabitha Johns and Ada Malcolm.

Contributors TOB, GG, ZW, BAB, AB, MSS, SDA, CL, AMM, PAE, LLM, FAM and SCB contributed to study design. TJL, JCM and TOB contributed to manuscript composition. LLM, FAM and SCB provided critical revisions.

Funding This work was supported by P50 GM111152-01 awarded by the National Institute of General Medical Sciences (NIGMS). TJL, JCM and JAS were supported by a postgraduate training grant (T32 GM-08721) in burns, trauma and perioperative injury from the NIGMS.

Competing interests None declared.

Patient consent Obtained.

Ethics approval Approved by University of Florida Institutional Review Board.

Provenance and peer review Not commissioned; externally peer reviewed.

Open Access This is an Open Access article distributed in accordance with the Creative Commons Attribution Non Commercial (CC BY-NC 4.0) license, which permits others to distribute, remix, adapt, build upon this work non-commercially, and license their derivative works on different terms, provided the original work is properly cited and the use is non-commercial. See: http://creativecommons.org/ licenses/by-nc/4.0/

(C) Article author(s) (or their employer(s) unless otherwise stated in the text of the article) 2017. All rights reserved. No commercial use is permitted unless otherwise expressly granted.

\section{REFERENCES}

1. Yeh RW, Sidney S, Chandra M, et al. Population trends in the incidence and outcomes of acute myocardial infarction. $N$ Engl $J$ Med 2010;362:2155-65.

2. Torio CM, Andrews RM. National Inpatient Hospital Costs: The Most Expensive Conditions by Payer, 2011: Statistical Brief \#160. Healthcare Cost and Utilization Project (HCUP) Statistical Briefs. Rockville, 2006.

3. Angus DC, Linde-Zwirble WT, Lidicker J, et al. Epidemiology of severe sepsis in the United States: analysis of incidence, outcome, and associated costs of care. Crit Care Med 2001;29:1303-10.

4. Kaukonen KM, Bailey M, Pilcher D, et al. Systemic inflammatory response syndrome criteria in defining severe sepsis. N Engl J Med 2015;372:1629-38.

5. Gentile LF, Cuenca AG, Efron PA, et al. Persistent inflammation and immunosuppression: a common syndrome and new horizon for surgical intensive care. J Trauma Acute Care Surg 2012;72:1491-501.

6. Rosenthal MD, Moore FA. Persistent inflammatory, immunosuppressed, catabolic syndrome (PICS): A new phenotype of multiple organ failure. J Adv Nutr Hum Metab 2015;1.

7. Croft CA, Moore FA, Efron PA, et al. Computer versus paper system for recognition and management of sepsis in surgical intensive care. J Trauma Acute Care Surg 2014;76:311-7.
8. McKinley BA, Moore LJ, Sucher JF, et al. Computer protocol facilitates evidence-based care of sepsis in the surgical intensive care unit. J Trauma 2011;70:1153-67.

9. Sucher JF, Moore FA, Todd SR, et al. Computerized clinical decision support: a technology to implement and validate evidence based guidelines. J Trauma 2008;64:520-37.

10. Gardner-Thorpe J, Love N, Wrightson J, et al. The value of Modified Early Warning score (MEWS) in surgical in-patients: a prospective observational study. Ann R Coll Surg Engl 2006;88:571-5.

11. Muckart DJ, Bhagwanjee S. American College of chest Physicians/ Society of Critical Care Medicine Consensus Conference definitions of the systemic inflammatory response syndrome and allied disorders in relation to critically injured patients. Crit Care Med 1997;25:1789-95.

12. Levy MM, Fink MP, Marshall JC, et al. 2001 SCCM/ESICM/ACCP/ ATS/SIS International Sepsis Definitions Conference. Crit Care Med 2003;31:1250-6.

13. Davies MG, Hagen PO. Systemic inflammatory response syndrome. Br J Surg 1997;84:920-35

14. Dellinger RP, Levy MM, Rhodes A, et al. Surviving sepsis campaign: international guidelines for management of severe sepsis and septic shock: 2012. Crit Care Med 2013;41:580-637.

15. Nathens AB, Johnson JL, Minei JP, et al. Inflammation and the host response to Injury, a large-scale collaborative project: patientoriented research core--standard operating procedures for clinical care. I. guidelines for mechanical ventilation of the trauma patient. $J$ Trauma 2005;59:764-9.

16. Minei JP, Nathens AB, West M, et al. Inflammation and the Host response to Injury, a Large-Scale Collaborative Project: patientoriented research core-standard operating procedures for clinical care. II. guidelines for prevention, diagnosis and treatment of ventilator-associated pneumonia (VAP) in the trauma patient. $J$ Trauma 2006;60:1106-13.

17. Moore FA, McKinley BA, Moore EE, et al. Inflammation and the Host response to Injury, a large-scale collaborative project: patient-oriented research core--standard operating procedures for clinical care. III. guidelines for shock resuscitation. J Trauma 2006;61:82-9.

18. West MA, Shapiro $M B$, Nathens $A B$, et al. Inflammation and the host response to injury, a large-scale collaborative project: patientoriented research core-standard operating procedures for clinical care. IV. guidelines for transfusion in the trauma patient. J Trauma 2006;61:436-9.

19. Shapiro $M B$, West $M A$, Nathens $A B$, et al. V. guidelines for sedation and analgesia during mechanical ventilation general overview. J Trauma 2007;63:945-50.

20. Harbrecht BG, Minei JP, Shapiro MB, et al. Inflammation and the host response to injury, a large-scale collaborative project: patientoriented research core-standard operating procedures for clinical care: vi. blood glucose control in the critically ill trauma patient. $J$ Trauma 2007;63:703-8.

21. West MA, Moore EE, Shapiro MB, et al. Inflammation and the host response to injury, a large-scale collaborative project: patientoriented research core--standard operating procedures for clinical care VII--guidelines for antibiotic administration in severely injured patients. J Trauma 2008;65:1511-9.

22. O'Keefe GE, Shelton M, Cuschieri J, et al. Inflammation and the host response to injury, a large-scale collaborative project: patientoriented research core--standard operating procedures for clinical care VIII--Nutritional support of the trauma patient. J Trauma 2008;65:1520-8.

23. Evans HL, Cuschieri J, Moore EE, et al. Inflammation and the host response to injury, a Large-Scale Collaborative Project: patientoriented research core standard operating procedures for clinical care IX. Definitions for complications of clinical care of critically injured patients. J Trauma 2009;67:384-8.

24. Silver GM, Klein MB, Herndon DN, et al. Standard operating procedures for the clinical management of patients enrolled in a prospective study of inflammation and the Host response to thermal Injury. J Burn Care Res 2007;28:222-30.

25. Ely EW, Margolin R, Francis J, et al. Evaluation of delirium in critically ill patients: validation of the Confusion Assessment Method for the Intensive Care Unit (CAM-ICU). Crit Care Med 2001;29:1370-9.

26. Sessler CN, Gosnell MS, Grap MJ, et al. The Richmond AgitationSedation Scale: validity and reliability in adult intensive care unit patients. Am J Respir Crit Care Med 2002;166:1338-44.

27. Rahman A, Hasan RM, Agarwala R, et al. Identifying critically-il patients who will benefit most from nutritional therapy: Further validation of the 'modified NUTRIC' nutritional risk assessment tool. Clin Nutr 2016;35:158-62. 
28. Singer M, Deutschman CS, Seymour CW, et al. The Third International Consensus Definitions for Sepsis and Septic shock (Sepsis-3). JAMA 2016;315:801-10.

29. Finkelsztein EJ, Jones DS, Ma KC, et al. Comparison of qSOFA and SIRS for predicting adverse outcomes of patients with suspicion of sepsis outside the intensive care unit. Crit Care 2017;21:73.

30. Harris PA, Taylor R, Thielke R, et al. Research electronic data capture (REDCap)--a metadata-driven methodology and workflow process for providing translational research informatics support. J Biomed Inform 2009;42:377-81.

31. Mansur A, Hinz J, Hillebrecht B, et al. Ninety-day survival rate of patients with sepsis relates to programmed cell death 1 genetic polymorphism rs11568821. J Investig Med 2014;62:638-43.

32. Anton SD, Manini TM, Milsom VA, et al. Effects of a weight loss plus exercise program on physical function in overweight, older women: a randomized controlled trial. Clin Interv Aging 2011;6:141-9.

33. 2012 best practices for repositories collection, storage, retrieval, and distribution of biological materials for research international society for biological and environmental repositories. Biopreserv Biobank 2012;10:79-161.

34. von Elm E, Altman DG, Egger M, et al. The strengthening the Reporting of Observational studies in Epidemiology (STROBE) statement: guidelines for reporting observational studies. PLoS Med 2007;4:e296.

35. Jones AE, Trzeciak S, Kline JA. The sequential organ failure Assessment score for predicting outcome in patients with severe sepsis and evidence of hypoperfusion at the time of emergency department presentation. Crit Care Med 2009;37:1649-54.

36. Knaus WA, Draper EA, Wagner DP, et al. APACHE II: a severity of disease classification system. Crit Care Med 1985;13:818-29.

37. Hays RD, Sherbourne CD, Mazel RM. The RAND 36-Item Health Survey 1.0. Health Econ 1993;2:217-27.

38. Kaiser MJ, Bauer JM, Ramsch C, et al. Validation of the Mini Nutritional Assessment short-form (MNA-SF): a practical too for identification of nutritional status. J Nutr Health Aging 2009;13:782-8.

39. EuroQol G. EuroQol--a new facility for the measurement of healthrelated quality of life. Health Policy 1990;16:199-208.

40. Rasmusson DX, Bylsma FW, Brandt J. Stability of performance on the Hopkins Verbal Learning Test. Arch Clin Neuropsychol 1995;10:21-6.

41. Lezak MD. Neuropsychological assessment. 3rd ed. New York: Oxford University Press, 1995

42. McDowell I, Kristjansson B, Hill GB, et al. Community screening for dementia: the Mini Mental State Exam (MMSE) and modified Mini-Mental state exam (3MS) compared. J Clin Epidemiol 1997:50:377-83.

43. Oken MM, Creech RH, Tormey DC, et al. Toxicity and response criteria of the Eastern Cooperative Oncology Group. Am J Clin Oncol 1982;5:649-56.

44. Guralnik JM, Simonsick EM, Ferrucci L, et al. A short physical performance battery assessing lower extremity function: association with self-reported disability and prediction of mortality and nursing home admission. J Gerontol 1994;49:M85-M94.

45. Mitsiopoulos N, Baumgartner RN, Heymsfield SB, et al. Cadaver validation of skeletal muscle measurement by magnetic resonance imaging and computerized tomography. $J$ Appl Physiol 1998;85:115-22.

46. Mourtzakis M, Prado CM, Lieffers JR, et al. A practical and precise approach to quantification of body composition in cancer patients using computed tomography images acquired during routine care. Appl Physiol Nutr Metab 2008;33:997-1006.

47. Mira JC, Gentile LF, Mathias BJ, et al. Sepsis pathophysiology, chronic critical illness, and persistent InflammationImmunosuppression and catabolism syndrome. Crit Care Med 2017;45:253-62. 